\title{
IP Optics Measurement in the Coupled Situation
}

\author{
Y. Luo and D. Trbojevic
}

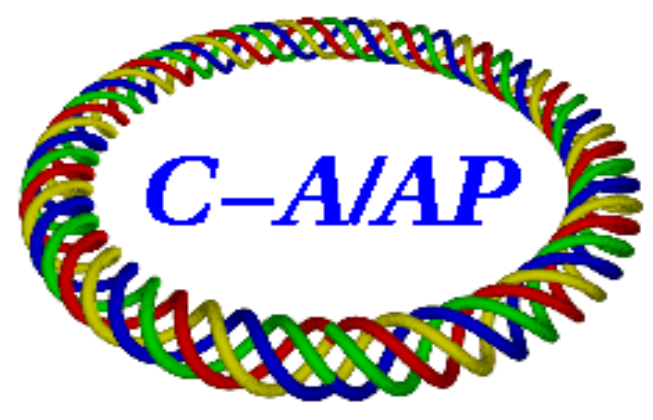

Collider-Accelerator Department Brookhaven National Laboratory Upton, NY 11973 
$\mathrm{CAD} / \mathrm{AP} / 188$

January 26,2005

\title{
IP optics measurement in the coupled situation
}

\author{
Y. Luo, D. Trbojevic \\ Brookhaven National Laboratory, Upton, NY 11973, USA
}

The accelerator collision luminosity is inversely proportional to the betatron amplitude function $\beta^{*}$ at the collision point. To achieve higher luminosity, $\beta^{*}$ squeezing is the general solution if the physical and the dynamic apertures are big enough. Therefore, $\beta^{*}$ measurement plays an important role in the colliders.

In this AP note, we present methods to measure the beam optics at the interaction point, in the coupled situation. Emphasis is put to the measurements of the $\beta^{\star}, \beta$ waist, coupling parameters. The linear coupling's action-angle parameterization is used here. And the basic assumption is that there are two dual-plane BPMs on both sides of the interaction point, and between them there is no magnet.

\section{Introduction}

In the uncoupled situation, the conventional method to determine the $\beta^{*}$ is to measure the $\beta$ at the first quadrupole to the interaction point (IP). If there is a $\Delta k$ change in the first quadrupole strength, the tune shifts are

$$
\left\{\begin{array}{rl}
\Delta \mu_{x} & =\frac{1}{4 \pi} \bar{\beta}_{x} \Delta k d l \\
\Delta \mu_{y} & =-\frac{1}{4 \pi} \bar{\beta}_{y} \Delta k d l
\end{array},\right.
$$

where $\bar{\beta}_{x}$ and $\bar{\beta}_{y}$ are the horizontal and vertical averages of the $\beta$ functions in the quadrupole, $d l$ is the length of the quadrupole.

If there is no any magnet between the first quadrupole and the IP, the $\beta$ function at the first quadrupole can be analytically described in the $\beta^{*}$ and the distance $s$ between them,

$$
\beta(s)_{x, y}=\beta_{x, y}^{*}+\frac{s^{2}}{\beta_{x, y}^{*} .}
$$

Here we assume that the $\beta$ waist, where $\alpha_{x, y}^{*}=0$, is at the IP. Combining Eq. (2) and Eq. (1), the $\beta_{x, y}^{*}$ can be derived. To increase the measurement resolution, the quadrupole strength modulation is used, where a phase lock loop (PLL) system is used to track the fast tune modulations.

The above method assumes: 1) there is no magnet between the IP and the first quadrupole, 2) at IP, $\left.\alpha_{x, y}^{*}=0,3\right)$ the distance between the first quadrupole and the IP is known, 4)no any betatron coupling in the accelerator.

In each interaction region (IR) of the RHIC, there are two DXBPMs which are close to the last separation magnet DX and facing the interaction point (IP). If one ignores detector magnets or just switches it, the turn-by-turn angle informations $x^{\prime}$ and $y^{\prime}$ at the two BPMs can be obtained through $x$ and $y$ readings at these two DXBPMs, together with their separation distance.

In this article, we demonstrate methods to extract Twiss and coupling parameters if the turn-by-turn $\left(x, x^{\prime}, y, y^{\prime}\right)$ data are known at the DXBPMs, based on the linear coupling's action-angle parameterization. The $\beta$ waist and the $\beta$ function there can derived from the Twiss parameters at the DXBPMs. The $\beta^{*}$ can also be obtained from the turn-by-turn $\left(x, x^{\prime}, y, y^{\prime}\right)$ data at the $\beta$ waist. A fast method to check the $\beta^{*}$ from the phase advance between the two DXBPMs is presented.

\section{Linear coupling's action-angle parameterization [1]}

Here we quickly review the necessary ingredients of the linear coupling's action-angle parameterization for the following Twiss and coupling parameters' measurements. 


\subsection{Turn-by-turn coordinates}

For general two-dimensional linearly coupled motion, single-particle motion is

$$
\left(\begin{array}{c}
x \\
x^{\prime} \\
y \\
y^{\prime}
\end{array}\right)=\mathbf{P}\left(\begin{array}{c}
\sqrt{2 J_{I}} \cos \Phi_{I} \\
-\sqrt{2 J_{I}} \sin \Phi_{I} \\
\sqrt{2 J_{I I}} \cos \Phi_{I I} \\
-\sqrt{2 J_{I I}} \sin \Phi_{I I}
\end{array}\right)
$$

where $J_{I, I I}$ are the constant actions of the two eigen modes and $\Phi_{I, I I}$ are the eigen mode phases. One-turn phase advances for the two eigen modes are $2 \pi \mu_{I, I I}$. Therefore, the coordinates in the laboratory frame are

$$
\left\{\begin{array}{rl}
x & =p_{11} \sqrt{2 J_{I}} \cos \Phi_{I}+p_{13} \sqrt{2 J_{I I}} \cos \Phi_{I I}-p_{14} \sqrt{2 J_{I I}} \sin \Phi_{I I} \\
x^{\prime} & =p_{21} \sqrt{2 J_{I}} \cos \Phi_{I}-p_{22} \sqrt{2 J_{I}} \sin \Phi_{I}+p_{23} \sqrt{2 J_{I I}} \cos \Phi_{I I}-p_{24} \sqrt{2 J_{I I}} \sin \Phi_{I I} \\
y & =p_{31} \sqrt{2 J_{I}} \cos \Phi_{I}-p_{32} \sqrt{2 J_{I}} \sin \Phi_{I}+p_{33} \sqrt{2 J_{I I}} \cos \Phi_{I I} \\
y^{\prime} & =p_{41} \sqrt{2 J_{I}} \cos \Phi_{I}-p_{42} \sqrt{2 J_{I}} \sin \Phi_{I}+p_{43} \sqrt{2 J_{I I}} \sin \Phi_{I I}-p_{44} \sqrt{2 J_{I I}} \sin \Phi_{I I}
\end{array} .\right.
$$

If the turn-by-turn $\left(x, x^{\prime}, y, y^{\prime}\right)$ data at one point are known experimentally, $\mathbf{P}$ can be constructed.

\subsection{Matrix $\mathrm{P}$ and Twiss, coupling parameters}

$\mathbf{P}$ can be described in Twiss and coupling parameters [2],

$$
\mathbf{P}=\left(\begin{array}{cccc}
r \sqrt{\beta_{I}} & 0 & c_{11} \sqrt{\beta_{I I}}-\frac{c_{12} \alpha_{I I}}{\sqrt{\beta_{I I}}} & -\frac{c_{12}}{\sqrt{\beta_{I I}}} \\
-\frac{\alpha_{I} r}{\sqrt{\beta_{I}}} & \frac{r}{\sqrt{\beta_{I}}} & c_{21} \sqrt{\beta_{I I}}-\frac{c_{22} \alpha_{I I}}{\sqrt{\beta_{I I}}} & \frac{c_{22}}{\sqrt{\beta_{I I}}} \\
-\frac{c_{12} \alpha_{I}}{\sqrt{\beta_{I}}}-c_{22} \sqrt{\beta_{I}} & \frac{c_{12}}{\sqrt{\beta_{I}}} & r \sqrt{\beta_{I I}} & 0 \\
\frac{c_{11} \alpha_{I}}{\sqrt{\beta_{I}}}+c_{21} \sqrt{\beta_{I}} & -\frac{c_{11}}{\sqrt{\beta_{I}}} & -\frac{\alpha_{I I} r}{\sqrt{\beta_{I I}}} & \frac{r}{\sqrt{\beta_{I I}}}
\end{array}\right) .
$$

Inversely, Twiss and coupling parameters can be obtained from $\mathbf{P}$, too.

$$
\begin{gathered}
\left\{\begin{aligned}
\beta_{I} & =\frac{p_{11}}{p_{22}} \\
\alpha_{I} & =-\frac{p_{21}}{p_{22}} \\
\gamma_{I} & =\frac{1+\alpha_{I}^{2}}{\beta_{I}}=\frac{p_{21}^{2}+p_{22}^{2}}{p_{11} p_{22}}
\end{aligned}\right. \\
\left\{\begin{aligned}
\beta_{I I} & =\frac{p_{33}}{p_{44}} \\
\alpha_{I I} & =-\frac{p_{43}}{p_{44}} \\
\gamma_{I I} & =\frac{1+\alpha_{I I}^{2}}{\beta_{I I}}=\frac{p_{43}^{2}+p_{44}^{2}}{p_{33} p_{44}} \\
r & =\sqrt{p_{11} p_{22}}=\sqrt{p_{33} p_{44}}, \\
\mathbf{C} & =\mathbf{P}_{12} \mathbf{U}_{2}^{-1}=r \mathbf{P}_{12} \mathbf{P}_{22}^{-1} .
\end{aligned}\right.
\end{gathered}
$$

\subsection{Propagation of $\mathrm{P}$}

Defining

$$
\mathbf{G}=\mathbf{T}_{1 \rightarrow 2} \mathbf{P}_{1}
$$

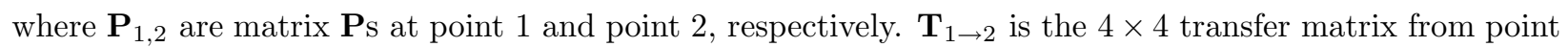
1 to point 2. Twiss parameters at point $s_{2}$ can be obtained from $\mathbf{G}$.

$$
\left\{\begin{array}{c}
\widetilde{\beta_{I}}=\frac{G_{11}^{2}+G_{12}^{2}}{G_{11} G_{22}-G_{12} G_{21}} \\
\widetilde{\alpha_{I}}=-\frac{G_{11} G_{21}+G_{12} G_{22}}{G_{11} G_{22}-G_{12} G_{21}}
\end{array},\right.
$$




$$
\left\{\begin{array}{l}
\widetilde{\beta_{I I}}=\frac{G_{33}^{2}+G_{34}^{2}}{G_{33} G_{44}-G_{34} G_{43}} \\
\widetilde{\alpha_{I I}}=-\frac{G_{33} G_{43}+G_{34} G_{44}}{G_{33} G_{44}-G_{34} G_{43}}
\end{array} .\right.
$$

The coupling parameter $\tilde{r}$ at $s_{2}$ is given by

$$
\tilde{r}=\sqrt{\widetilde{p_{11}} \widetilde{p_{22}}}=\sqrt{G_{11} G_{22}-G_{12} G_{21}},
$$

or

$$
\tilde{r}=\sqrt{\widetilde{p_{33} \widetilde{p_{44}}}}=\sqrt{G_{33} G_{44}-G_{34} G_{43}} .
$$

The phase advances between the two points are

$$
\begin{gathered}
\Delta \Phi_{I}=\arctan \frac{G_{12}}{G_{11}}, \\
\Delta \Phi_{I I}=\arctan \frac{G_{34}}{G_{33}} .
\end{gathered}
$$

\section{Experimental determinating of IP optics}

Here we give one example of Twiss and coupling parameter measurements. And both eigen mode motions are assumed. We will come back later to discuss only one eigen motion, which is a special case to the general treatment.

\subsection{Two BPMs in a drift}

The two DXBPMs in IR8 in the Blue ring of RHIC are considered. The design distance from the two BPMs to the design IP is $8.33 \mathrm{~m}$. We designate the DXBPM in the upstream of IP8 as g7-bx, and the DXBPM in the downstream of IP8 as g8-bx. We assume that there is no other magnet between the two DXBPMs. Knowing the turn-by-turn position data $(x, y)$ at the two DXBPMs, their turn-by-turn angles are

$$
\left\{\begin{array}{l}
x_{1,2}^{\prime}=\frac{x_{2}-x_{1}}{2 L} \\
y_{1,2}^{\prime}=\frac{y_{2}-y_{1}}{2 L}
\end{array},\right.
$$

where $z_{1}$ and $z_{2}, z=x$ or $y$, are the position readings at the g7-bx and g8-bx, respectively. $2 L$ is the distance between them. The angles $x^{\prime}$ and $y^{\prime}$ are same at the two DXBPMs.

\subsection{Eigen tunes}

In the following the simulation code $\mathrm{SAD}[3]$ is used to produce the turn-by-turn $(x, y)$ data at these two DXBPMs. In the simulation, only one free oscillation particle circulates in the Blue ring. The uncoupled tunes are $\mu_{x, 0}=28.22, \mu_{y, 0}=29.23$.

The skew quadrupole family 1 's integrated strength is set to be $0.0005 \mathrm{~m}^{-1}$ to introduce coupling into the uncoupled accelerator model. From the FFT of $(x+y)$ turn-by-turn data at g7-bx, the two coupled tunes are obtained $28.2126,29.2375$, respectively.

\subsection{Initial phases}

Using harmonic analysis, the initial phase $\phi_{I, 0}$ for eigen mode I at one point is

$$
\left\{\begin{aligned}
\phi_{I, 0} & =\arctan \left(\frac{-S_{I}}{C_{I}}\right) \\
C_{I} & =\sum_{i=1}^{N} x_{i} \cos \left[2 \pi \mu_{I}(n-1)\right] \\
S_{I} & =\sum_{i=1}^{N} x_{i} \sin \left[2 \pi \mu_{I}(n-1)\right]
\end{aligned}\right.
$$

$N$ is the maximum data-taking turn. 
Similarly, the initial phase $\phi_{I I, 0}$ for eigen mode II is

$$
\left\{\begin{array}{rl}
\phi_{I I, 0} & =\arctan \left(\frac{-S_{I I}}{C_{I I}}\right) \\
C_{I I} & =\sum_{i=1}^{N} y_{i} \cos \left[2 \pi \mu_{I I}(n-1)\right] \\
S_{I I} & =\sum_{i=1}^{N} y_{i} \sin \left[2 \pi \mu_{I I}(n-1)\right]
\end{array} .\right.
$$

From turn-by-turn BPM tracking data at g7-bx, eigen mode I and II's initial phases are $138.6449^{\circ}$ and $-152.1502^{\circ}$ respectively. At g8-bx, eigen mode I and II's initial phases are $305.0668^{\circ}$ and $14.0882^{\circ}$, respectively, so the phase advances between the two BPMs are $166.4219^{\circ}$ and $166.2384^{\circ}$ for eigen mode I

and II, respectively. From an analytical calculation with the simulation code SAD, they are $166.4212^{\circ}$ and $166.2399^{\circ}$, respectively.

\subsection{Transition matrix F}

Eq. (3) can be re-written as

$$
\begin{gathered}
\left(\begin{array}{c}
x \\
x^{\prime} \\
y \\
y^{\prime}
\end{array}\right)=\mathbf{F}\left(\begin{array}{c}
\cos \Phi_{I} \\
\sin \Phi_{I} \\
\cos \Phi_{I I} \\
\sin \Phi_{I I}
\end{array}\right), \\
\mathbf{F}=\left(\begin{array}{cccc}
p_{11} \sqrt{J_{I}} & 0 & p_{13} \sqrt{J_{I I}} & -p_{14} \sqrt{J_{I I}} \\
p_{21} \sqrt{J_{I}} & -p_{22} \sqrt{J_{I}} & p_{23} \sqrt{J_{I I}} & -p_{24} \sqrt{J_{I I}} \\
p_{31} \sqrt{J_{I}} & -p_{32} \sqrt{J_{I}} & p_{33} \sqrt{J_{I I}} & 0 \\
p_{41} \sqrt{J_{I}} & -p_{42} \sqrt{J_{I}} & p_{43} \sqrt{J_{I I}} & -p_{44} \sqrt{J_{I I}}
\end{array}\right) .
\end{gathered}
$$

F can be obtained from turn-by-turn $\left(x, x^{\prime}, y, y^{\prime}\right)$ data. For example,

$$
\left\{\begin{array}{l}
F_{11}=\frac{2}{N} \sum_{i=1}^{N} x_{i} \cos \left[2 \pi \mu_{I}(n-1)+\phi_{I, 0}\right] \\
F_{12}=\frac{2}{N} \sum_{i=1}^{N} x_{i} \sin \left[2 \pi \mu_{I}(n-1)+\phi_{I, 0}\right] \\
F_{13}=\frac{2}{N} \sum_{i=1}^{N} x_{i} \cos \left[2 \pi \mu_{I I}(n-1)+\phi_{I I, 0}\right] \\
F_{14}=\frac{2}{N} \sum_{i=1}^{N} x_{i} \sin \left[2 \pi \mu_{I I}(n-1)+\phi_{I I, 0}\right]
\end{array} .\right.
$$

Similarly, from $x^{\prime}, y$ and $y^{\prime}$ turn-by-turn data, $F_{2 j}, F_{3 j}$ and $F_{4 j}, j=1,2,3,4$ can be obtained.

From the above tracking data at g7-bx, we obtain

$$
\mathbf{F}=\left(\begin{array}{cccc}
7.505 \times 10^{-4} & -1.852 \times 10^{-16} & -1.451 \times 10^{-4} & 5.040 \times 10^{-5} \\
-8.898 \times 10^{-5} & -1.061 \times 10^{-5} & 1.790 \times 10^{-5} & -3.912 \times 10^{-6} \\
4.579 \times 10^{-4} & 1.675 \times 10^{-4} & 2.390 \times 10^{-4} & 4.485 \times 10^{-17} \\
-5.187 \times 10^{-5} & -2.634 \times 10^{-5} & -2.831 \times 10^{-5} & -3.419 \times 10^{-6}
\end{array}\right)
$$

\subsection{Obtain matrix $\mathrm{P}$}

Matrix $\mathbf{F}$ includes action information. The ratio of the two actions is

$$
k=\sqrt{\frac{J_{I}}{J_{I I}}}=\sqrt{\frac{\left\|\mathbf{F}_{11}\right\|}{\left\|\mathbf{F}_{22}\right\|}} .
$$

According to the definition of matrix $\mathbf{F}$, the signs of the elements in its second and fourth columns are inverted, and the first two column elements are divided by $k$. Normalizing the new matrix's determinant to be 1, we get the matrix $\mathbf{P}$ at g7-bx,

$$
\mathbf{P}=\left(\begin{array}{cccc}
6.873 & 1.696 \times 10^{-12} & -4.385 & -1.523 \\
-0.815 & 0.097 & 0.541 & 0.118 \\
4.193 & -1.534 & 7.225 & -1.356 \times 10^{-12} \\
-0.475 & 0.241 & -0.856 & 0.103
\end{array}\right)
$$




\subsection{Twiss and coupling parameters}

Knowing $\mathbf{P}$ at g7-bx, Twiss and coupling parameters there can be calculated, according to Eqs. (6) - (9),

$$
\begin{aligned}
& r=\frac{\sqrt{p_{11} p_{22}}+\sqrt{p_{33} p_{44}}}{2}=\frac{8.42+8.38}{2}=0.840, \\
& \left\{\begin{array}{l}
\beta_{I}=70.86 \\
\alpha_{I}=8.40
\end{array},\right. \\
& \left\{\begin{array}{l}
\beta_{I I}=70.15 \\
\alpha_{I I}=8.31
\end{array},\right. \\
& \mathbf{C}=\left(\begin{array}{cc}
-1.981 & -12.421 \\
0.177 & 0.962
\end{array}\right) \text {. }
\end{aligned}
$$

And one can prove that here $r^{2}+\|\mathbf{C}\|=0.996$.

\section{$3.7 \beta$ waist and $\beta^{*}$}

The transfer matrix for the drift from the IP to the DXBPM is

$$
\mathbf{T 1 2}=\left(\begin{array}{cccc}
1 & L & 0 & 0 \\
0 & 1 & 0 & 0 \\
0 & 0 & 1 & L \\
0 & 0 & 0 & 1
\end{array}\right)
$$

$L$ is the distance from the $\beta$ waist to the DXBPM. According to Eq. (10),

$$
\mathbf{G}=\mathbf{T 1 2} \cdot \mathbf{P 1}=\left(\begin{array}{cccc}
p_{11}+L \cdot p_{21} & L \cdot p_{22} & p_{13}+L \cdot p_{23} & p_{14}+L \cdot p_{24} \\
p_{21} & p_{22} & p_{23} & p_{24} \\
p_{31}+L \cdot p_{41} & p_{32}+L \cdot p_{42} & p_{33}+L \cdot p_{43} & L \cdot p_{44} \\
p_{41} & p_{42} & p_{43} & p_{44}
\end{array}\right)
$$

where $p_{i j}$ s are elements of $\mathbf{P}$ at IP. At the $\beta$ waist, $\alpha_{1,2}=0$, that is, $p_{21}=0, p_{43=0}$. Therefore, we get

$$
\mathbf{G}=\left(\begin{array}{cccc}
p_{11} & L \cdot p_{22} & p_{13}+L \cdot p_{23} & p_{14}+L \cdot p_{24} \\
0 & p_{22} & p_{23} & p_{24} \\
p_{31}+L \cdot p_{41} & p_{32}+L \cdot p_{42} & p_{33} & L \cdot p_{44} \\
p_{41} & p_{42} & 0 & p_{44}
\end{array}\right)
$$

Twiss parameters at the DXBPMs are

$$
\begin{aligned}
& \left\{\begin{array}{l}
\widetilde{\beta_{I}}=\frac{p_{11}^{2}+\left(L \cdot p_{22}\right)^{2}}{p_{11} p_{22}}=\beta_{I}^{*}+L^{2} / \beta_{I}^{*}, \\
\widetilde{\alpha_{I}}=-L \cdot \beta_{I}^{*}
\end{array}\right. \\
& \left\{\begin{array}{l}
\widetilde{\beta_{I I}}=\frac{p_{33}^{2}+\left(L \cdot p_{44}\right)^{2}}{p_{33} p_{44}}=\beta_{I I}^{*}+L^{2} / \beta_{I I}^{*} . \\
\widetilde{\alpha_{I I}}=-L \cdot \beta_{I I}^{*}
\end{array}\right.
\end{aligned}
$$

According to Eq. (33) and the Twiss parameters at DXBPM, we can determine where the eigen mode I's $\beta$ waist is and the $\beta_{1}$ there. For simplicity, we designate the $\beta$ s at the waist as $\beta^{*}$ s.

$$
\left\{\begin{array}{rl}
\beta_{I}^{*} & =\frac{\widetilde{\beta_{I}}}{1+{\widetilde{\alpha_{I}}}^{2}} \\
L_{I} & =\alpha_{I} * \beta_{I}^{*}
\end{array} .\right.
$$

Similarly, from Eq. (34), or eigen mode II,

$$
\left\{\begin{aligned}
\beta_{I I}^{*} & =\frac{\widetilde{\beta_{I I}}}{1+{\widetilde{\alpha_{I I}}}^{2}} \\
L_{I I} & =\alpha_{I I} * \beta_{I I}^{*}
\end{aligned}\right.
$$


$L_{I}$ and $L_{I I}$ are the distances from the eigen mode I and eigen mode II's waists to DXBPMs, respectively.

Based on Twiss parameters obtained at g7-bx in the above, we easily get the $\beta^{*}$ and the $\beta$ waist for each eigen modes. For eigen mode I,

$$
\left\{\begin{array}{l}
\beta_{I}^{*}=0.99 \\
L_{I}=8.317
\end{array}\right.
$$

For eigen mode II,

$$
\left\{\begin{array}{l}
\beta_{I I}^{*}=1.00 \\
L_{I I}=8.31
\end{array} .\right.
$$

Actually, knowing where the $\beta$ waist is, the turn-by-turn $\left(x, x^{\prime}, y, y^{\prime}\right)$ data there can be constructed from these at the two DXBPMs. So the Twiss and coupling parameters can also be calculated from $\mathbf{P}$ there. Of course, the Twiss and coupling parameters at the $\beta$ waist also can be calculated through $\mathbf{P}$ 's propagation [1].

\subsection{Fast check $\beta^{*}$ from the phase advance}

With high performance digital beam position monitor (BPM) and modern analytical techniques $[4,5,6,7]$, phases and phase advances for the two eigen modes can be measured very precisely. The merit of the phase measurement is that it is insensitive to BPM offsets and gains. In the following, we propose a possible method to fast check $\beta^{*}$. This method is decided by the resolution of the phase measurement.

According to Eq. (31) and Eq. (18), Eq. (19), the eigen mode phase advances between the $\beta$ waist to one DXBPM are

$$
\left\{\begin{array}{c}
\Delta \phi_{I}=\arctan \left(L \cdot p_{22} / p_{11}\right)=\arctan \left(L / \beta_{I}^{*}\right) \\
\Delta \phi_{I I}=\arctan \left(L \cdot p_{44} / p_{33}\right)=\arctan \left(L / \beta_{I I}^{*}\right)
\end{array} .\right.
$$

If we assume the IP locates at the center of the interaction regeion (IR), the total phase advances between the two DXBPMs are

$$
\left\{\begin{array}{rl}
\Delta \phi_{I}(\text { dxbpms }) & =2 \arctan \left(L / \beta_{I}^{*}\right) \\
\Delta \phi_{I I}(\text { dxbpms }) & =2 \arctan \left(L / \beta_{I I}^{*}\right)
\end{array},\right.
$$

where $\Delta \phi_{I, I I}(d x b p m s)$ are the total phase advances between the two DXBPMs.

Knowing the phase advance between the two DXBPMs, $\beta^{*}$ s can be derived.

$$
\left\{\begin{array}{l}
\beta_{I}^{*}=\frac{L}{\tan \frac{\Delta \phi_{I}(d x b p m s)}{2}} \\
\beta_{I I}^{*}=\frac{L}{\tan \frac{\Delta \phi_{I I}(d x b p m s)}{2}}
\end{array} .\right.
$$

Fig. 1 shows the phase advances between the two DXBPMs for different $\beta^{*}$, where the distance of the DXBPMs are assumed to be $8.33 \times 2 \mathrm{~m}$.

\subsection{One eigen mode excitation}

If only one eigen mode is activated, we still can obtain the $\mathbf{F}$ from the turn-by-turn $\left(x, x^{\prime}, y, y^{\prime}\right)$ at the two DXBPMs. The only difference compared to the above free oscillation is that there is only one eigen tune motion in the horizontal or vertical plane. For only eigen mode I activating,

$$
\mathbf{F}=\left(\begin{array}{cccc}
p_{11} \sqrt{J_{I}} & 0 & 0 & 0 \\
p_{21} \sqrt{J_{I}} & -p_{22} \sqrt{J_{I}} & 0 & 0 \\
p_{31} \sqrt{J_{I}} & -p_{32} \sqrt{J_{I}} & 0 & 0 \\
p_{41} \sqrt{J_{I}} & -p_{42} \sqrt{J_{I}} & 0 & 0
\end{array}\right) .
$$

For only eigen mode II excitation,

$$
\mathbf{F}=\left(\begin{array}{cccc}
0 & 0 & p_{13} \sqrt{J_{I I}} & -p_{14} \sqrt{J_{I I}} \\
0 & 0 & p_{23} \sqrt{J_{I I}} & -p_{24} \sqrt{J_{I I}} \\
0 & 0 & p_{33} \sqrt{J_{I I}} & 0 \\
0 & 0 & p_{43} \sqrt{J_{I I}} & -p_{44} \sqrt{J_{I I}}
\end{array}\right) .
$$

Then we revert the signs of the elements $F_{i j} \mathrm{~s}$ in the second columns and the fourth columns of $\mathbf{F}$, and only normalize the matrix $\mathbf{F}_{11}$ or $\mathbf{F}_{22}$ 's determinant to 1 for the eigen mode I or eigen mode II activating, 


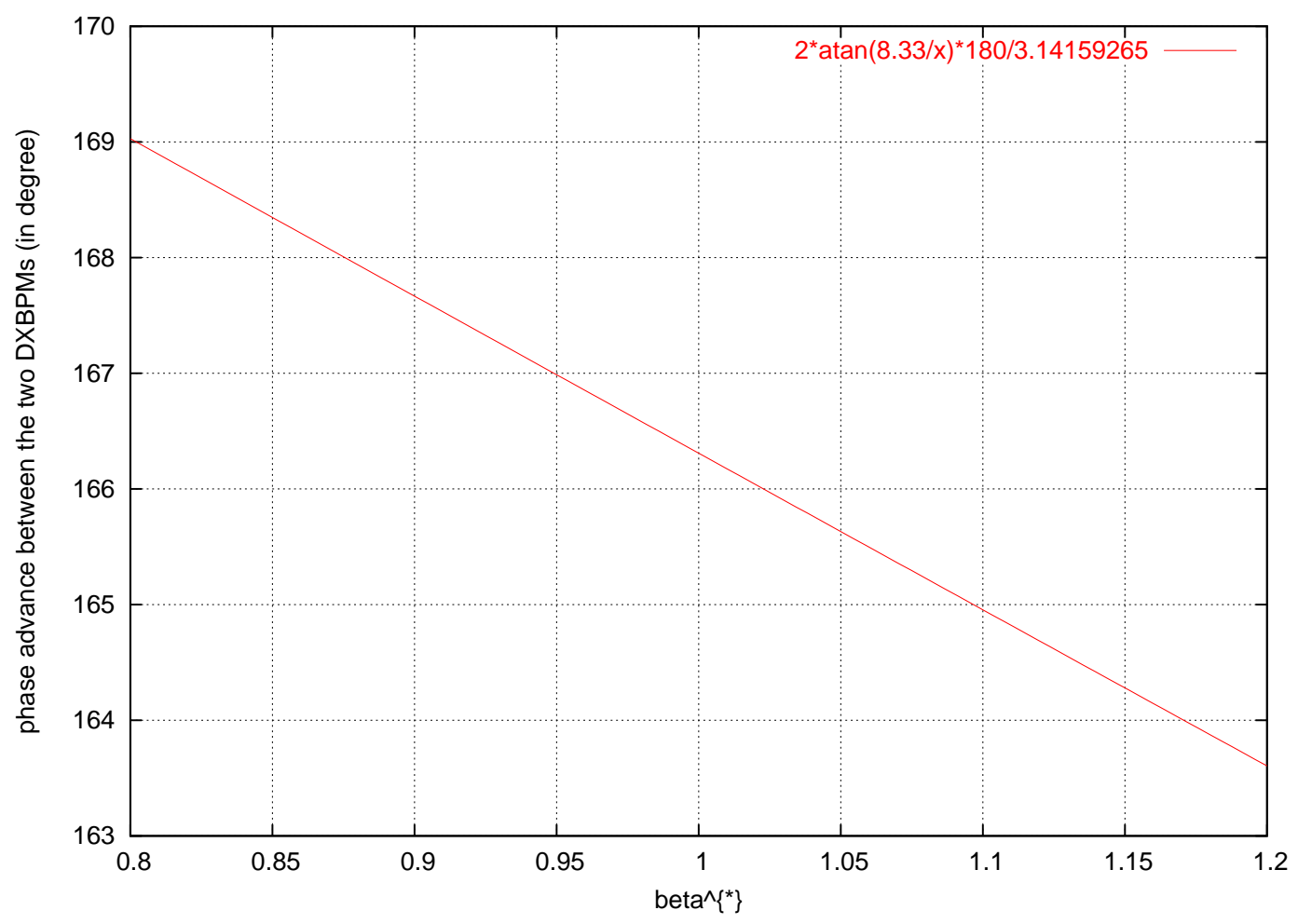

Figure 1: The phase advances between the two DXBPMs for different $\beta^{*}$.

respectively. The Twiss functions for eigen mode I or eigen mode II are calculated from $\mathbf{P}_{11}$ or $\mathbf{P}_{22}$, according to Eqs. (6) - (7). Knowing eigen mode I's Twiss functions and $\mathbf{P}_{21}$ at one DXBPM, the coupling matrix $\mathbf{C}$ can be determined. Knowing the eigen mode II's Twiss functions and matrix $\mathbf{P}_{12}$ at one DXBPM, the coupling matrix $\mathbf{C}$ can be determined, too.

\section{Acknowledgments}

The authors would like to thank T. Satogata, V. Ptisyn, M. Bai for discussions. This work is support by the US DOE under contract No. DE-AC02-98CH10886.

\section{References}

[1] Y. Luo, Linear coupling's parameterization in the rotation frame, RHIC-AP-165, 2004.

[2] D. Sagan and D. Rubin, Phys. Rev. ST Accel. Beam 2, 074001 (1999)

[3] SAD code homepage: http://acc-physics.kek.jp/SAD/sad.html .

[4] D. Sagan, Phys. Rev. ST Accel. Beam 4, 122801 (2001).

[5] D. Sagan et al., Phys. Rev. ST Accel. Beam 3, 102801 (2000).

[6] J. Irwin et al., Phys. Rev. Lett. 82, p.1684, 1999.

[7] C.-x. Wang et al., Phys. Rev. ST Accel. Beams 6, 104001(2003) 